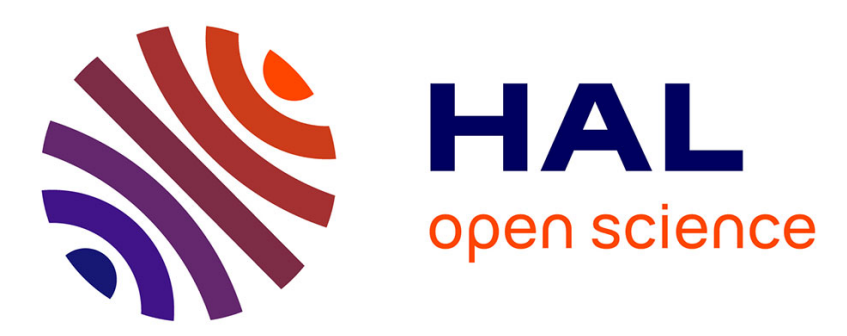

\title{
On modelling brick/mortar interface via a St. Venant-Kirchhoff orthotropic soft interface. Part II: in silico analysis
}

\author{
Maria Letizia Raffa, Frédéric Lebon, Raffaella Rizzoni
}

\section{- To cite this version:}

Maria Letizia Raffa, Frédéric Lebon, Raffaella Rizzoni. On modelling brick/mortar interface via a St. Venant-Kirchhoff orthotropic soft interface. Part II: in silico analysis. International Journal of Masonry Research and Innovation, 2017, 2 (4), pp.259-273. 10.1504/IJMRI.2017.087430 . hal01694049

\section{HAL Id: hal-01694049 \\ https://hal.science/hal-01694049}

Submitted on 6 Jun 2018

HAL is a multi-disciplinary open access archive for the deposit and dissemination of scientific research documents, whether they are published or not. The documents may come from teaching and research institutions in France or abroad, or from public or private research centers.
L'archive ouverte pluridisciplinaire HAL, est destinée au dépôt et à la diffusion de documents scientifiques de niveau recherche, publiés ou non, émanant des établissements d'enseignement et de recherche français ou étrangers, des laboratoires publics ou privés. 


\title{
On modelling brick/mortar interface via a St. Venant-Kirchhoff orthotropic soft interface. Part II: in silico analysis
}

\author{
Maria Letizia Raffa* \\ CNRS, \\ Laboratoire Modélisation et Simulation Multi-Echelle, \\ UMR CNRS 8208, \\ 61, Av. du Général de Gaulle, \\ 94010 Créteil Cedex, \\ France \\ Email: maria-letizia.raffa@u-pec.fr \\ ${ }^{*}$ Corresponding author
}

\section{Frédéric Lebon}

\author{
Aix-Marseille University, \\ CNRS, \\ Centrale Marseille, \\ Laboratory of Mechanics and Acoustics, \\ 4 Impasse Nikola Tesla CS 40006 - 13453, \\ Marseille Cedex 13, \\ France \\ Email: lebon@1ma.cnrs-mrs.fr
}

\section{Raffaella Rizzoni}

Department of Engineering, University of Ferrara,

via Saragat 1,

44121 Ferrara,

Italy

Email: raffaella.rizzoni@unife.it

\begin{abstract}
A new strategy for the numerical modelling of brick/mortar interfaces at the macro-scale taking into account finite strains and evolving microcracking phenomena is introduced. A numerical validation of the non-linear-imperfect interface model formulated in Part I of the present paper, is proposed. Wellestablished experimental data concerning diagonal compression of masonry walls are simulated within the finite element method. The localisation zones of highest displacement jumps and stresses obtained through the numerical simulations are in good agreement with the experimental findings. The proposed non-linear interface model is also compared with a linear interface model for masonry structures.
\end{abstract}


Keywords: asymptotic analysis; damage; FEM; finite strain; imperfect interfaces; masonry structures; microcracking; micromechanical homogenisation; orthotropic interphase; spring-like models.

Reference to this paper should be made as follows: Raffa, M.L., Lebon, F. and Rizzoni, R. (2017) 'On modelling brick/mortar interface via a St. VenantKirchhoff orthotropic soft interface. Part II: in silico analysis', Int. J. Masonry Research and Innovation, Vol. 2, No. 4, pp.259-273.

Biographical notes: Maria Letizia Raffa, $\mathrm{PhD}$, is a Postdoctoral Researcher in the Modelling and Multiscale Simulation Laboratory of CNRS, Créteil, France. She received Master's degree in Civil Engineering from University of Messina and her Doctorate in joint supervision in Solid Mechanics from University of Rome 'Tor Vergata' and Aix-Marseille University, with a thesis entitled "Micromechanical modelling of imperfect interfaces and applications". Her current research interests include imperfect interface modelling with applications in civil engineering, biomechanics and contact mechanics.

Frédéric Lebon, $\mathrm{PhD}$, Hab is a full-time Professor of Solid Mechanics at AixMarseille University, France. He received his Master's degree and his PhD in Applied Mathematics in Marseille University and the Habilitation in Mechanics in Montpellier University. He is actually head of the Mechanics and Acoustics Laboratory in Marseille. His work focuses on computational mechanics, contact mechanics, interface modeling and non-smooth mechanics. He published more than 80 papers in international journals and more than 100 proceedings of conferences. His h-factor is 15 (Scopus)/21 (Scholar).

Raffaella Rizzoni, $\mathrm{PhD}$, is an Assistant Professor of Solid Mechanics at the University of Ferrara, Italy. She received the degree in Engineering of Materials at the University of Ferrara and her $\mathrm{PhD}$ in Structural Engineering at the University of Florence. Her research interests are in continuum mechanics, elasticity, thin structures and smart materials. Her research activity is documented by more than 50 papers, published in journals and proceedings of national and international conferences. Her actual h-index is 9 (source Scopus, January 2017).

\section{Introduction}

Recently, there has been a growing interest in micromodelling approaches for simulating the behaviour of masonry structures (Alfano and Sacco, 2006; Gabor et al., 2006b; Milani et al., 2006; Calderini and Lagomarsino, 2008; Pelissou and Lebon, 2009; Rekik and Lebon, 2010, 2012; Scimemi et al., 2014). In such approaches, the masonry joints are represented by mortar continuous elements coupled with discontinuous brick/mortar interface elements.

In Part I of the present study (Raffa et al., 2016a), a non-linear-imperfect interface model for brick/mortar interfaces was formulated. This model, hereinafter referred as the St. Venant-Kirchhoff (SVK) interface model, was obtained by coupling arguments of asymptotic analysis (Abdelmoula et al., 1998; Benveniste, 2006; Lebon and Rizzoni, 2008, 2010, 2011; Rizzoni and Lebon, 2013; Rizzoni et al., 2014), extended to the finite strain theory (Dumont et al., 2014; Rizzoni et al., 2017), with a homogenisation method for microcracked media under the non-interacting approximation (Kachanov, 1994; Mauge and Kachanov, 1994; Tsukrov and Kachanov, 2000; Sevostianov and Kachanov, 2013). 
This second part of the study proposes a numerical validation of the SVK interface model. The latter is implemented within a finite element analysis (FEA) in COMSOL Multiphysics ${ }^{\circledR}$ software, currently used in numerical simulations of masonry structures (Vermeltfoort and van Schijndel, 2008, 2010, 2013). Therefore, for comparison with the SVK interface model, a linear brick/mortar interface model following the spring-like model proposed by Rekik and Lebon $(2010,2012)$, in a revised version by Fouchal et al. (2014), is also implemented. In the numerical analyses concerning the masonry walls, either with linear and non-linear interface models, the brick and the mortar materials are both assumed to be linear elastic and isotropic materials. By using a preliminary standard homogenisation analysis in agreement with Rekik and Lebon (2012), the material parameters of both interface models are obtained from the material properties of a thin interphase, supposed to be a two-layered composite body sandwiched between the principal masonry constituents (cf. Appendix A in Rekik and Lebon (2012)).

The SVK interface model is briefly recalled in Section 2. In Section 3, the FEA is applied to simulate the tests of the experimental program carried out by Gabor et al. $(2006 \mathrm{a}, \mathrm{b})$ on masonry walls under diagonal compression. In the same section, the linear and the non-linear models, i.e. comprised of linear and non-linear interfaces, respectively, are compared in terms of the Von Mises stresses and shear stresses.

Finally, an original FEA analysis adopting a bilinear evolution law for the microcrack length, aiming to take into account a damaging phenomenon localised at the brick/masonry interface, is proposed. The evolution law depends on the imposed displacement and it is chosen in according with former work by the authors Rekik and Lebon (2012), Fouchal et al. (2014). The proposal and implementation of the SVK interface evolutive model are elements of novelty of the present paper.

\section{The St. Venant-Kirchhoff interface law}

This section recalls the basic elements of the non-linear-soft interface model formulated in Raffa et al. (2016a), which are mandatory to understand its numerical implementation within the FEA. For further details concerning the fundamentals of the SVK interface formulation, one can refer to former works (Raffa, 2015; Rizzoni et al., 2017; Raffa et al., 2016a).

The SVK interface model has been developed within the framework of the imperfect interface approach (IIA) (Raffa, 2015). The IIA allows to model, from a mechanical point of view, a microcracked thin interphase as a zero-thickness interface. To this aim, the IIA is employed to obtain the interface constitutive law. The approach consists in coupling, in a mathematically rigorous way, the matched asymptotic expansion (MAE) method (SanchezPalencia, 1980) and a micromechanical homogenisation theory for microcracked media (Kachanov, 1994; Mauge and Kachanov, 1994; Tsukrov and Kachanov, 2000; Sevostianov and Kachanov, 2013).

The MAE method extended to the finite strain theory (Rizzoni et al., 2017; Raffa et al., 2016a), particularly to the SVK elastic problem (Krasucki et al., 2001), allows to obtain the following soft-non-linear interface law:

$$
\begin{aligned}
& {[[\mathbf{P n}]]=\mathbf{0},} \\
& \mathbf{P n}=\frac{A_{\mathrm{NNNN}}^{\varepsilon}}{2 \varepsilon^{3}}|[[\mathbf{u}]]|^{2}[[\mathbf{u}]],
\end{aligned}
$$


in which $\mathbf{P}$ is the first Piola-Kirchhoff stress tensor, $\mathbf{n}$ is the unit normal vector characterising the interface, $\varepsilon$ is the interphase thickness and $[[\mathbf{u}]]=\left\{u_{\mathrm{T}} u_{\mathrm{N}}\right\}$ represents the displacement-jump vector at the interface, in which $\mathrm{N}$ and $\mathrm{T}$ denote the normal and tangential-to-the-interface directions, respectively.

As well established in solid mechanics, a soft-interface is characterised by a continuity of the stress vector Pn (Eq. (1)) and a jump in terms of displacements at the interface. This latter is usually expressed by a linear relationship, nevertheless it has been demonstrated (Rizzoni et al., 2017; Raffa et al., 2016a, 2017) that within the finite strain theory, a non-linear relationship between the stress vector and the displacement-jump vector arises (Eq. (2)). The stiffness coefficient $A_{\mathrm{NNNN}}^{\varepsilon}$, appearing in Eq. (2), is obtained by a micromechanical homogenisation analysis within the non-interacting approximation (Sevostianov and Kachanov, 2013; Mauge and Kachanov, 1994; Tsukrov and Kachanov, 2000) in a stress-based approach (Raffa, 2015). For further details about recovering the effective mechanical properties of a microcracked interphase, one can refer to former works (Sevostianov and Kachanov, 2013; Mauge and Kachanov, 1994; Tsukrov and Kachanov, 2000; Raffa, 2015).

Particularly, its final expression, in the case of initial orthotropic interphase weakened by one family of parallel microcracks (Raffa, 2015), reads as:

$$
A_{\mathrm{NNNN}}^{\varepsilon}=\frac{E_{\mathrm{T}} E_{\mathrm{N}}}{E_{\mathrm{T}}+2 \rho B_{\mathrm{N}} E_{\mathrm{T}} E_{\mathrm{N}}-E_{\mathrm{N}} \nu_{\mathrm{TN}}^{2}},
$$

with

$$
B_{\mathrm{N}}=\frac{\pi}{2 \sqrt{E_{\mathrm{N}}}}\left(\frac{2}{\sqrt{E_{\mathrm{T}} E_{\mathrm{N}}}}+\frac{1}{G_{\mathrm{TN}}}-\frac{2 \nu_{\mathrm{TN}}}{E_{\mathrm{T}}}\right)^{\frac{1}{2}},
$$

where $E_{\mathrm{T}}, E_{\mathrm{N}}, G_{\mathrm{TN}}$ and $\nu_{\mathrm{TN}}$ are the elastic constants of the undamaged orthotropic interphase, obtained following a preliminary homogenisation approach for layered composites similar to a previous work by Rekik and Lebon (2012).

In Eq. (3), the microcracks are taken into account through the microcrack density $\rho$, which form is $\rho=\frac{l^{3}}{V}$ in $3 \mathrm{D}$ problems and $\rho=\frac{l^{2}}{S}$ in $2 \mathrm{D}$, being $V$ (resp. $S$ ) the volume (resp. the surface) of the representative elementary domain (Kachanov, 1994; Mauge and Kachanov, 1994; Tsukrov and Kachanov, 2000; Rekik and Lebon, 2010, 2012; Raffa, 2015). Note also that the stiffness coefficient $A_{\mathrm{NNNN}}^{\varepsilon}$ satisfies the soft-interphase assumption of linearly rescaling with $\varepsilon$ (Geymonat et al., 1999). As a result, the stiffness coefficient $A_{\mathrm{NNNN}}^{\varepsilon}$ can be expressed as follows:

$$
A_{\mathrm{NNNN}}^{\varepsilon}=\varepsilon \hat{A}_{\mathrm{NNNN}} \simeq \varepsilon\left(\frac{L}{2 B_{\mathrm{N}} l^{2}}\right)
$$

in which $L$ is a geometric parameter representing a characteristic length for the interfaces and $l$ is the microcrack length, or equivalently the damage parameter. By substituting Eq. (5) into Eq. (2), this last can be rewritten as follows:

$$
\mathbf{P n}=\frac{\hat{A}_{\mathrm{NNNN}}}{2 \varepsilon^{2}}|[[\mathbf{u}]]|^{2}[[\mathbf{u}]]
$$


This latter expression for the non-linear interfaces is used in the following numerical simulations in order to model the brick/mortar interfaces in both principal directions of the masonry wall.

\section{In silico experiments: diagonal compression test on a masonry wall}

The brick/mortar interface behaviour at the macro-scale is investigated through FEA using the COMSOL Multiphysics ${ }^{\circledR}$ software. This kind of finite element method (FEM)based software is currently used in numerical simulations concerning masonry structures (Vermeltfoort and van Schijndel, 2008, 2010, 2013). In particular, reference is herein made to the experimental program carried out by Gabor et al. $(2006 \mathrm{a}, \mathrm{b})$ concerning diagonal compression test on masonry walls. In the following, the experimental tests, i.e. setup and main experimental findings, are briefly recalled. Next, the proposed numerical simulations are detailed and discussed.

\subsection{Experimental background}

Gabor and his co-authors focused their experimental study on in-plane shear behaviour of unreinforced and fiber-reinforced masonry panels (Gabor et al., 2006a,b). In the present study, only the experimental data concerning the unreinforced panels are considered. Each panel has nominal dimensions of $870 \times 840 \times 100 \mathrm{~mm}^{3}$ and it is composed of hollow bricks of dimensions $210 \times 100 \times 50 \mathrm{~mm}^{3}$ and regular vertical and horizontal mortar joints of thickness of $10 \mathrm{~mm}$. The material properties of the principal constituents, bricks and mortar, namely, have been evaluated by compression and shear tests on masonry prisms (Gabor et al., 2006a,b). The resulting mechanical properties, assuming an isotropic nature of both materials, are summarised in Table 1.

Table 1 Mechanical properties of the masonry constituents determined experimentally in Gabor et al. (2006a,b)

Bricks Young modulus (MPa)

Bricks Poisson ratio

$E_{b}=13 \times 10^{3}$

Mortar Young modulus (MPa)

$\nu_{b}=0.2$

Mortar Poisson ratio

$E_{m}=4 \times 10^{3}$

$\nu_{m}=0.2$

The masonry panel is subjected to a diagonal compression load within a quasi-static framework. The load is gradually applied to the up-right corner by a $500 \mathrm{kN}$ hydraulic jack and controlled through a load cell; moreover, the panel is opportunely fixed through a cemented shoe localised at the opposite corner (bottom-left) and the other corners are left free. According to these boundary conditions, the diagonal compression test ensures a combined state of shear and compression along the direction of the horizontal and vertical joints (Gabor et al., 2006a). The experimental setup for the diagonal compression test is schematically sketched in Figure 1. The test is performed in a load-controlled mode until failure. The displacements along compressed and stretched diagonal are measured by LVDT (linear variable differential transformer) transducers. 
Figure 1 Geometry and boundary conditions: synoptic sketch of the panel in diagonal compression studied by Gabor et al. (2006a,b). The fixed shoe and the hydraulic jack are schematically represented in blue and red, respectively, dimensions are expressed in millimeters (see online version for colours)

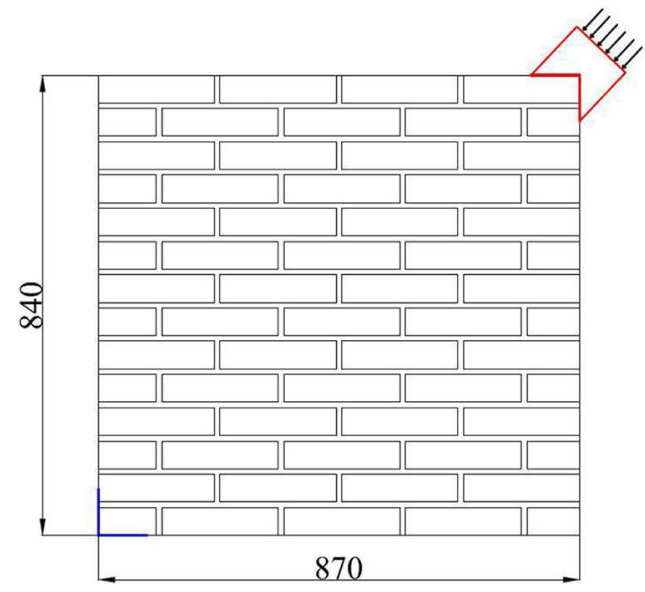

The experimental results (for further details one can refer to Gabor et al. (2006a,b)) highlighted that the unreinforced panels present a brittle failure along the compressed diagonal, with cracking phenomena that origin at the brick/mortar interfaces and evolve suddenly in the mortar joints and in the bricks, producing the instantaneous failure of the walls. Accordingly, the global behaviour, described by the applied load vs. strain curves and traced along the compressed diagonal, may be considered as quasi-elastic.

\subsection{Numerical simulation of Gabor's wall}

\subsubsection{A comparison between soft-linear and non-linear interfaces}

The proposed numerical simulation consists in a comparison between two numerical models of Gabor's wall in which two different interface laws have been implemented to model brick/mortar interfaces in both vertical and horizontal joints.

The first model, defined as non-linear, assumes non-linear SVK brick/mortar interfaces (Eq. (2)). The second model, called linear, assumes linear brick/mortar interfaces according to the spring-like model of Rekik-Lebon type (Rekik and Lebon, 2010, 2012; Fouchal et al., 2014; Raffa et al., 2016b). In particular, this last interface law

$$
\sigma \mathbf{n}=\mathbf{K}[[\mathbf{u}]]
$$

correlates the Cauchy-stress vector at the interface $\sigma \mathbf{n}$ to the displacement-jump vector $[[\mathbf{u}]]$, through the stiffness matrix $\mathbf{K}$, whose components depend on the microcracking features and on the mechanical characteristics of the undamaged material. The stiffness matrix K can be expressed as follows (Fouchal et al., 2014):

$$
\mathbf{K}=\operatorname{diag}\left[K_{\mathrm{T}} ; K_{\mathrm{N}}\right]=\operatorname{diag}\left[\frac{L}{B_{\mathrm{T}} l^{2}} ; \frac{L}{2 B_{\mathrm{N}} l^{2}}\right]
$$


where the parameter $B_{\mathrm{N}}$ is given by Eq. (4), and the parameter $B_{\mathrm{T}}$ is given by a similar expression (Kachanov, 1994) which reads as:

$$
B_{\mathrm{T}}=\frac{\pi}{2 \sqrt{E_{\mathrm{T}}}}\left(\frac{2}{\sqrt{E_{\mathrm{T}} E_{\mathrm{N}}}}+\frac{1}{G_{\mathrm{TN}}}-\frac{2 \nu_{\mathrm{TN}}}{E_{\mathrm{T}}}\right)^{\frac{1}{2}} .
$$

For both linear and non-linear models, brick and mortar materials are assumed to be linearly elastic and isotropic with the mechanical properties reported in Table 1. Note that in all proposed numerical models, the interphase is treated through the IIA. Thus, the $\varepsilon$-thick interphase consists in a third material supposed to be initially orthotropic. In particular, the interphase is assumed to be a thin stratified layer composed of brick and mortar localised at the brick/mortar interface level, i. e. sandwiched between bricks and mortar joints. Its material characteristics $E_{\mathrm{T}}, E_{\mathrm{N}}, G_{\mathrm{TN}}$ and $\nu_{\mathrm{TN}}$ are derived starting from the mechanical properties of the constituents $\left(E_{b}, E_{m}, \nu_{b}, \nu_{m}\right)$ by employing a preliminary standard homogenisation analysis of the undamaged $\varepsilon$-thick representative elementary volume (for more details, one can refer to Appendix A in Rekik and Lebon (2012)). Accordingly, the effective elastic moduli of the undamaged interphase material are $E_{\mathrm{N}}=$ $8.5 \times 10^{3} \mathrm{MPa}, E_{\mathrm{T}}=6.3 \times 10^{3} \mathrm{MPa}, G_{\mathrm{TN}}=5 \times 10^{3} \mathrm{MPa}$ and $\nu_{\mathrm{TN}}=0.2$.

Referring to the SVK interface model (Eq. (6)) and to the linear imperfect interface model of Rekik-Lebon type (Eq. (7)), the values of the interface stiffnesses result in: $\hat{A}_{3333}=5.9 \times 10^{4} \mathrm{~N} / \mathrm{mm}^{3}$ in the non-linear case and $K_{\mathrm{N}}=5.9 \times 10^{4} \mathrm{~N} / \mathrm{mm}^{3}$ and $K_{\mathrm{T}}=1.4 \times 10^{5} \mathrm{~N} / \mathrm{mm}^{3}$ in the linear case, respectively.

Both the linear (i. e. adopting a linear interface law) and the non-linear (i. e. adopting a non-linear interface law) numerical models of the wall in diagonal compression are implemented under the plane stress assumption. A free quadrangular mesh of quadratic Lagrangian order is chosen in both numerical cases. The brick/mortar interface is modelled through interface finite elements of zero thickness and both horizontal and vertical interfaces, following the masonry principal directions, are taken into account. In silico tests are performed in a displacement-controlled mode. Accordingly, the imposed displacement is incremented until its maximum value is reached. The maximum value of the imposed displacement is $3 \mathrm{~mm}$, and it is applied to the up-right corner through a rigid block which simulate the actuator, while the opposite corner is fixed. In order to accurately simulate the experimental setup, two rigid shoes in which a zero rotation is imposed are implemented on the secondary diagonal corners. The mesh of the masonry panel, consisting of about 7500 finite elements, is illustrated in Figure 2, where the panel deformation at the final loading step is also shown.

It is worth noting that the choice of performing the quasi-static simulations in displacement-controlled mode is made to avoid convergence problems in the solution, mostly in the non-linear case. As a consequence, the global force is derived as a reaction force on the top boundary of the block simulating the actuator .

A comparison between linear and non-linear models in terms of the Von Mises stresses and the shear stresses is shown in Figures 3 and 4, respectively. It is worth highlighting that the non-linear model is able to reproduce the localisation of stresses at the brick/mortar interfaces. This strong localisation is well established by experimental evidences (Gabor et al., 2006b). Moreover, the non-linear model seems to be globally less stiff than the linear one. Figures 3 and 4 show that, in the non-linear model, a stresses redistribution localises at the brick/mortar horizontal and vertical interfaces, particularly along the compressed diagonal. In contrast, in the linear case, the stresses are more uniformly distributed in the 
overall domain. The stress distribution predicted by the non-linear model can be considered a more realistic response for masonry panels than the one predicted by the linear case.

Figure 2 Mesh and deformation of the masonry panel - Sketch of the plane quadrangular mesh in the whole analysed system (on the left side) and panel's deformation at the final loading step, with a contour plot of the displacement field, expressed in millimeters (on the right hand side, see online version for colours)
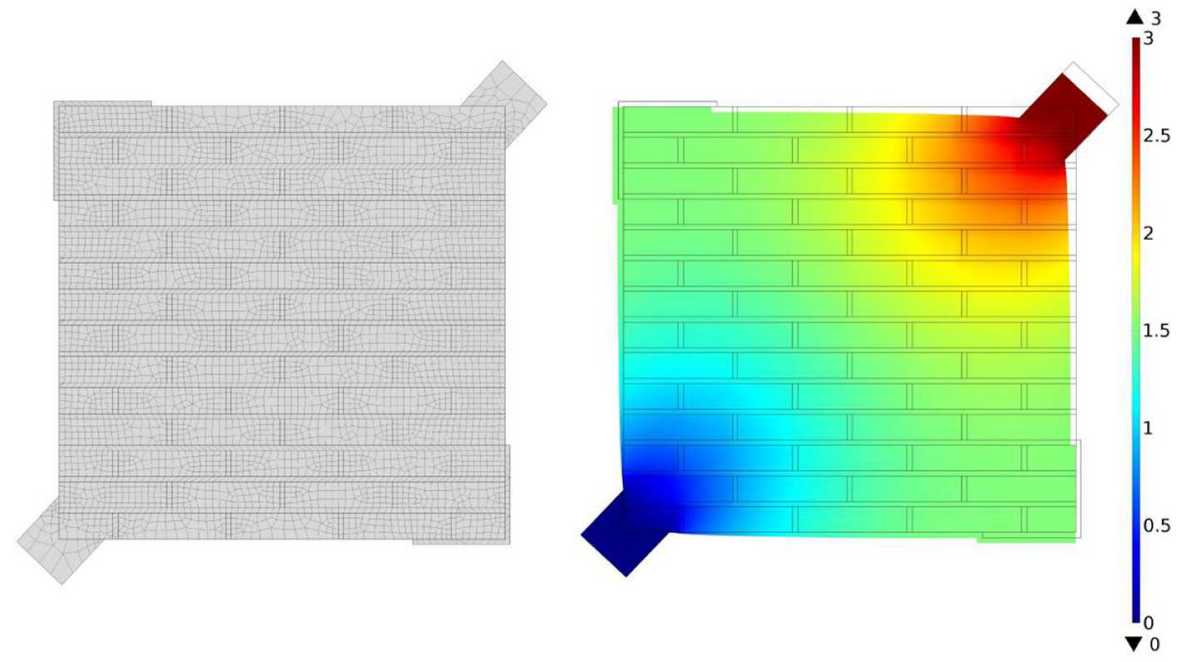

Most notably, the two numerical models implemented so far do not take into account an evolutive microcracking feature. Indeed, the microcrack equivalent length $l$ (Eqs. (5) and (8)) is assumed to be fixed in time and equal to $0.5 \mathrm{~mm}$, for both horizontal and vertical interfaces. In the next section, the introduction of a simple bilinear evolution law for $l$ will allow to investigate this phenomenon.

\subsubsection{Introduction of an evolutive microcracking}

The present section is devoted to investigate an evolving microcracking phenomenon localised at the brick/mortar interfaces. For this purpose, only the non-linear model (i.e. the masonry panel with non-linear interface condition) is taken into account.

The considered model is exactly the same as described above, with the same geometry, material properties, boundary conditions in terms of displacement and non-linear interface properties as in Eqs. (3)-(6). The novelty is the introduction of an evolution law for the microcrack length $l$ (cf. Eq. (5)) with respect to the imposed displacement. A bilinear law, as illustrated in Figure 5, is chosen according to Rekik and Lebon (2012), Fouchal et al. (2014).

The microcrack length $l$ is assumed to have the constant value $l_{e}=0.5 \mathrm{~mm}$ until the masonry panel reaches the elastic limit and then it linearly increases to the ultimate value $l_{u}=1 \mathrm{~mm}$ (refer to Figure 5), which is directly related to the ultimate strength of the masonry wall. The law parameters $l_{e}$ and $l_{u}$ are model-dependent, thus they are strictly related to the masonry panel behaviour. This fact can represent a shortcoming of 
the proposed model. Nevertheless, it is well established from an experimental point of view that masonry, due to its nature of hand-made material, does not present a standardised global behaviour, on the contrary, the experimental results are always much dispersed. To the best of the authors' knowledge, no models of masonry with a pure predictive nature of the damaging phenomena are available in structural analysis.

Figure 3 Von Mises stresses in MPa - Contour plot of the Von Mises stress field in the linear model (upward) and in the non-linear model (beneath), a factor scale of 10 is applied to the deformation (see online version for colours)

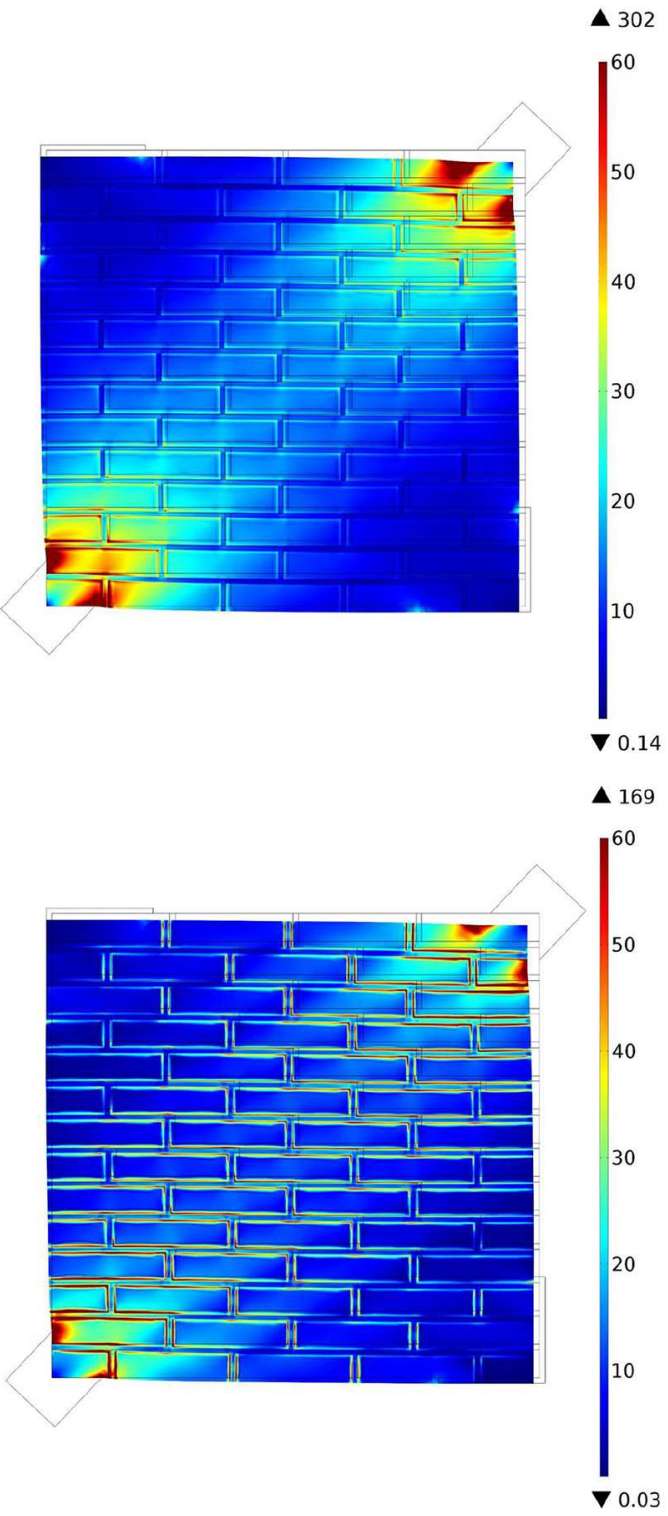


Given the above evolution law, a quasi-static simulation has also been performed. Figure 6 shows the distribution of the Von Mises stresses in the overall panel at the final increment step. The zoom allows to visualise the newly formed 'cracks' localised at the brick/mortar interfaces. These cracks are obviously related to the jump of the displacement vector prescribed by the implemented non-linear interface law in Eq. (6). Figure 6 also highlights that stresses are smaller than in the non-linear model with a fixed microcrack length (Figure 3); this can be explained with the rapid increase in the parameter $l$ once reached the elastic limit.

Figure 4 Shear stresses in MPa - Contour plot of the shear stress field in the linear model (upward) and in the non-linear model (beneath), a factor scale of 10 is applied to the deformation (see online version for colours)
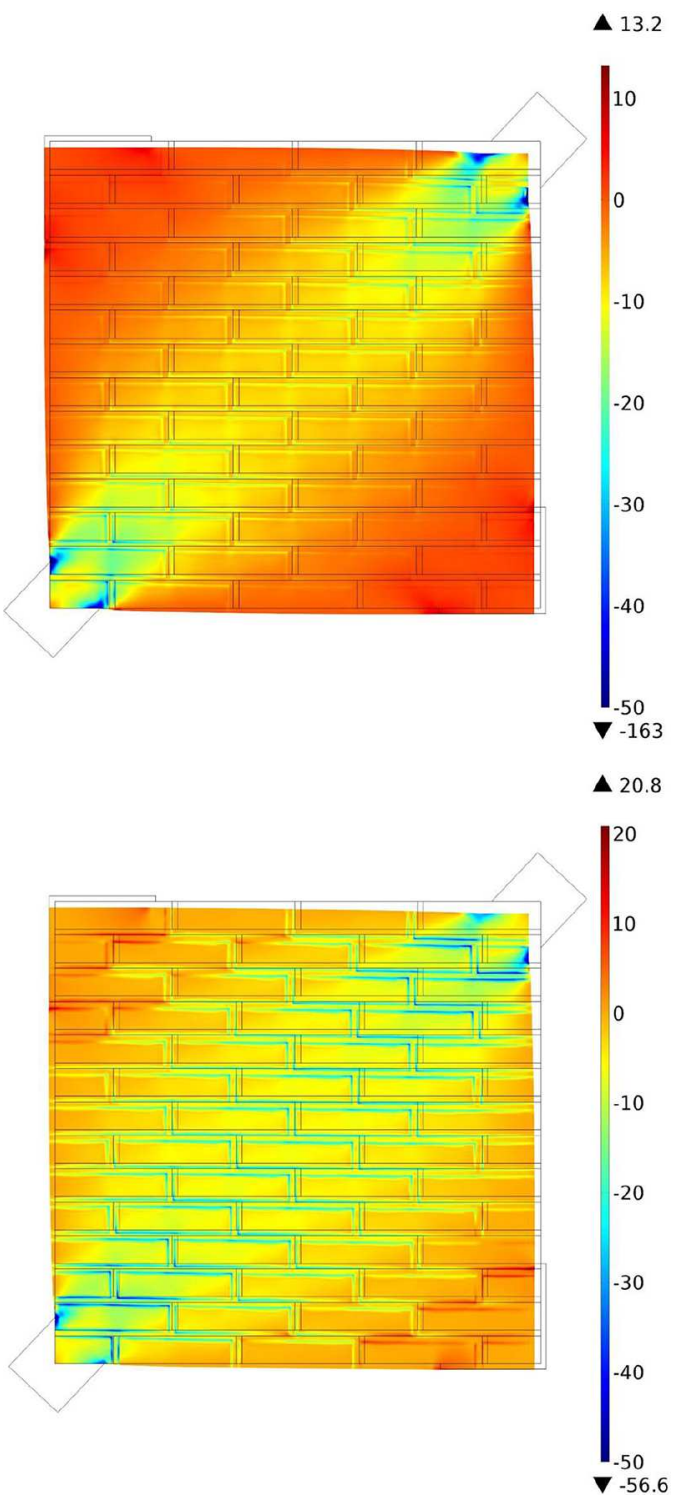
Figure 5 Evolution law - Bilinear evolution law of the microcrack length $l$ with respect to the imposed displacement $x$, adopted for the proposed simulation (see online version for colours)

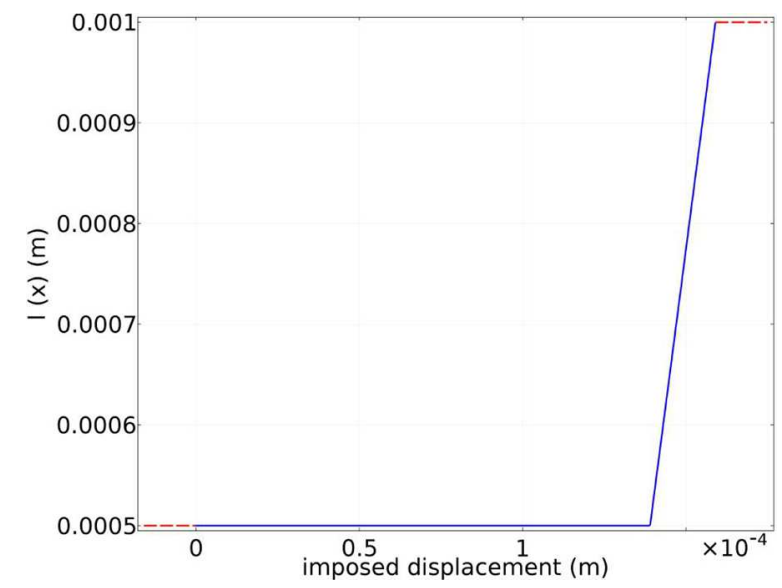

Figure 6 Von Mises stress in MPa - Contour plot of the Von Mises stress in the non-linear model with a detail of the zone near to the applied load (upper-right corner), highlighting the microcracking phenomenon at the brick/mortar interfaces (red arrows, see online version for colours)

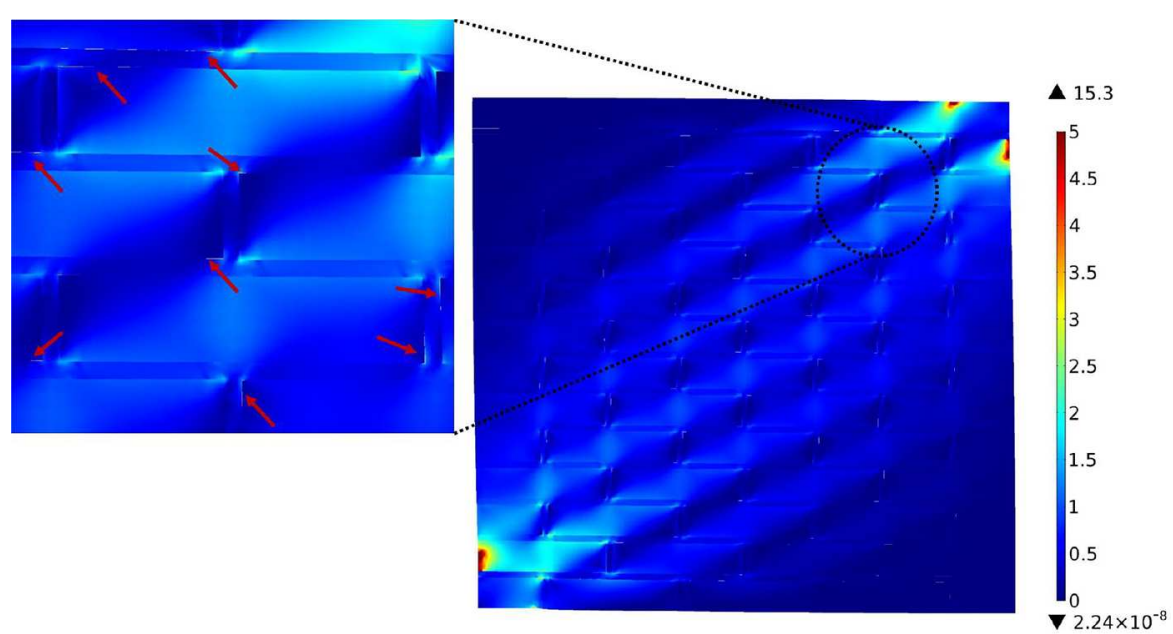

The displacement jumps at the brick/mortar interfaces are shown in Figure 7. As expected from some experimental evidences (Gabor et al., 2006a,b), the largest displacement jumps, corresponding to the developing of cracks at the brick/mortar interfaces, are localised along the compressed diagonal and, in particular, near the loaded corner, that is near the actuator imposing the load (at the upper-right corner) and the fixed shoe (at the bottom-left corner). The magnitude of the displacement jumps at the vertical interfaces appears to be larger than at the horizontal interfaces. This fact can be related to our simplified hypothesis of considering the same microcrack length evolution law for both kinds of interfaces. However, this simplifying assumption could be easily dropped. 
Figure 7 Displacement jump vector - Vector field visualisation of the displacement jump vector at both horizontal interfaces (upward, in red) and vertical interfaces (beneath, in blue), the arrows' dimension being proportional to the displacement jump magnitude (see online version for colours)
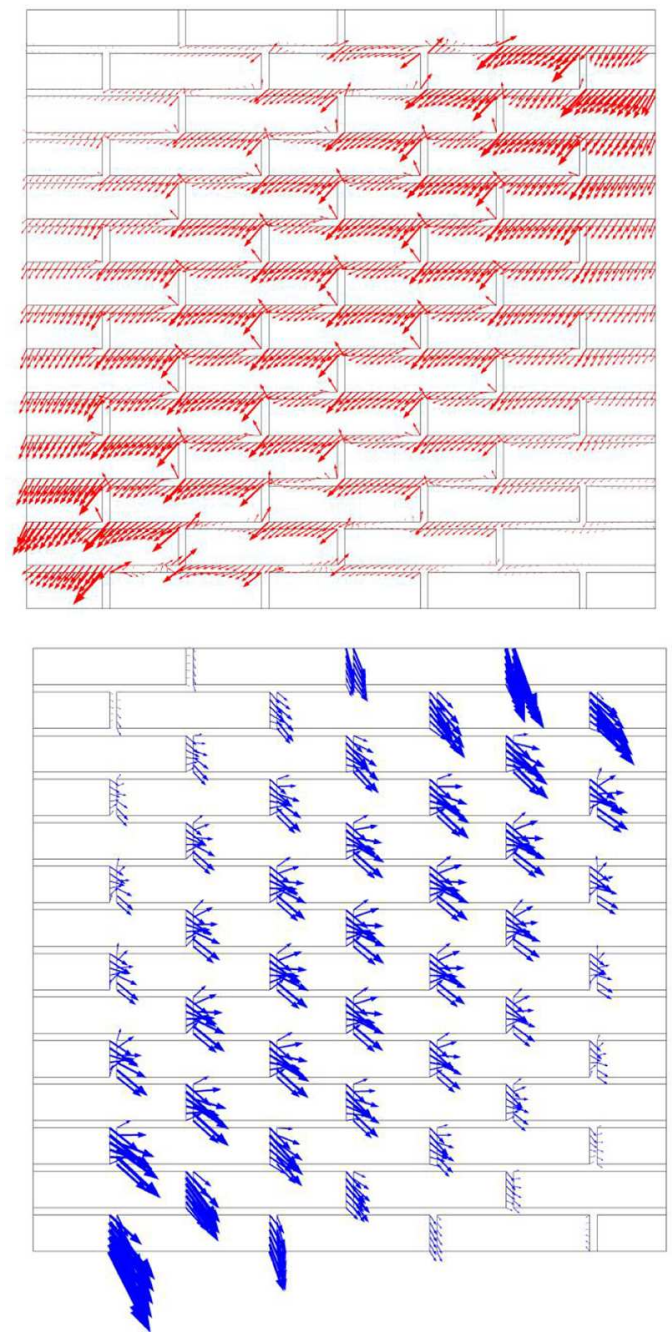

Finally, an influence analysis on the evolution-law parameter $l_{u}$ has been performed in terms of the wall's global behaviour. In particular, three values have been tested: $l_{u}=$ $\{1,5,10\} \mathrm{mm}$, and the resulting curves are shown in Figure 8. This last result highlights that the proposed brick/mortar interface model is able to take into account the post-peak behaviour, i.e. the damaging range after the elastic limit.

The model parameter $l_{u}$ governs this last behaviour. For the value $l_{u}=10 \mathrm{~mm}$, some rupture mechanisms are activated in the simulation, which we intend to further investigation in a next study. Nevertheless, this result shows the predictive potential of such a simplified damaged interface model. 
Figure 8 Global behaviour of wall in diagonal compression - the reaction force in the loaded-diagonal direction is plotted against the imposed displacement. Three values of the model parameter $l_{u}$ are tested (see online version for colours)

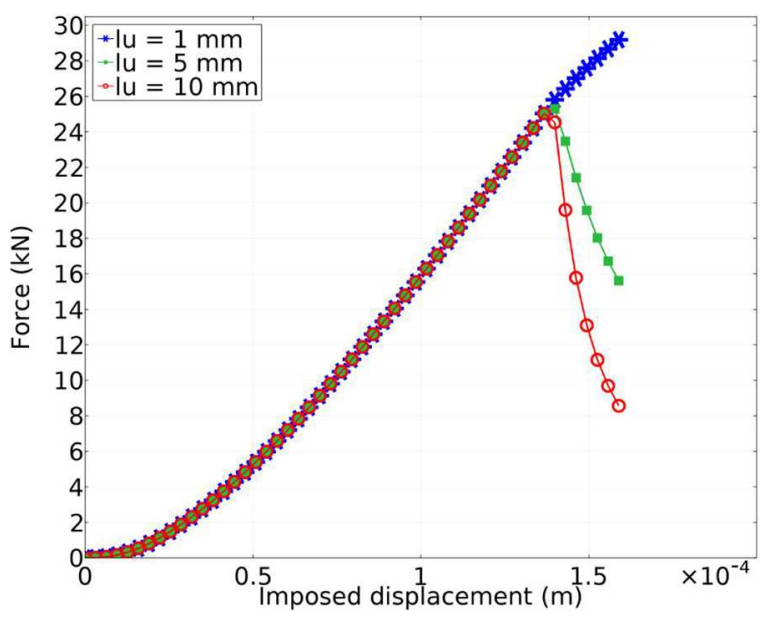

As already mentioned, the value $l_{e}=0.5 \mathrm{~mm}$ chosen in the previous simulations corresponds to an initial damage state localised at the brick/mortar interfaces. This explains the initial non-linear behaviour for low values of the imposed displacement in Figure 8 and suggests that a small value of the parameter $l_{e}$ has to be taken in order to observe a perfect elastic behaviour during the initial stage.

\section{Conclusion}

In the present paper, the brick/mortar interface behaviour at the macro-scale is simulated through the introduction of linear and non-linear interface models accounting for microcracking. The possibility of an evolutive behaviour of this phenomenon has also been introduced. Some finite element analyses have been proposed using COMSOL Multiphysics ${ }^{\circledR}$ software. Particularly, the experimental tests carried out by Gabor et al. $(2006 \mathrm{a}, \mathrm{b})$ concerning masonry walls under diagonal compression are simulated. The FEA indicates that the non-linear interface model provides a realistic description of the behaviour of the masonry structures; indeed, it is capable of reproducing the stress localisation experimentally observed at the brick/mortar interfaces.

The introduction of an evolutive law of the microcracking phenomenon represents an element of novelty of the paper. The evolution law, chosen as bilinear with respect to the imposed displacement, allows to reproduce the developing of cracks localised along the compressed diagonal, a fact which is currently observed in experiments on masonry panels under diagonal compression. Moreover, it is worth noting that the proposed interface model introduces only two model parameters. Nevertheless, we agree that a general technical results cannot clearly been derived from the present paper, due to the particular complex nature of the masonry material. However, we expect that the model herein presented can contribute to highlight the important role played by interfaces in masonry applications, which cannot be neglected or underestimated in technical applications. 
As a perspective of this research, the introduction of a more sophisticated point-wise damaging law, including a real dependency of $l$ on time, is in progress. In particular, an energetic relationship governing the time evolution of the damage parameter (crack length) $\dot{l}$ can be found through an asymptotic approach within the finite strain theory of the St. Venant-Kirchhoff type. This kind of law has already been implemented by authors in FEM analyses and successfully compared with experimental data concerning walls in shear test (Raffa et al., 2017).

\section{References}

Abdelmoula, R., Coutris, M. and Marigo, J.J. (1998) 'Comportement asymptotique d'une interface mince', Comptes Rendus de l'Académie des Sciences-Series IIB, Vol. 326, No. 4, pp.237-242.

Alfano, G. and Sacco, E. (2006) 'Combining interface damage and friction in a cohesivezone model', International Journal for Numerical Methods in Engineering, Vol. 68, No. 5, pp.542-582.

Benveniste, Y. (2006) 'An $O\left(h^{N}\right)$ interface model of a three-dimensional curved interphase in conduction phenomena', Proceedings of the Royal Society of London A, Vol. 462, pp.1593-1617.

Calderini, C. and Lagomarsino, S. (2008) 'Continuum model for in-plane anisotropic inelastic behavior of masonry', Journal of Structural Engineering-Asce, Vol. 134, No. 2, pp.209-220.

Dumont, S., Lebon, F. and Rizzoni, R. (2014) 'An asymptotic approach to the adhesion of thin stiff films', Mechanics Research Communications, Vol. 58, pp.24-35.

Fouchal, F., Lebon, F., Raffa, M.L. and Vairo, G. (2014) 'An interface model including cracks and roughness applied to masonry', The Open Civil Engineering Journal, Vol. 8, pp.263-271.

Gabor, A., Bennani, A., Jacquelin, E. and Lebon, F. (2006) 'Modelling approaches of the in-plane shear behaviour of unreinforced and FRP strengthened masonry panels', Composite Structures, Vol. 74, pp.277-288.

Gabor, A., Ferrier, E., Jacquelin, E. and Hamelin, P. (2006) 'Analysis of the in-plane shear behaviour of FRP reinforced hollow brick masonry walls', Construction and Building Materials, Vol. 20, pp.308-321.

Geymonat, G., Krasucki, F. and Lenci, S. (1999) 'Mathematical analysis of a bonded joint with a soft thin adhesive', Mathematics and Mechanics of Solids, Vol. 4, No. 2, pp.201-225.

Kachanov, M. (1994) 'Elastic solids with many cracks and related problems', Advances in Applied Mechanics, Vol. 30, pp.259-445.

Krasucki, F., Münch, A. and Ousset, Y. (2001) 'Asymptotic analysis of a bonded joint in nonlinear elasticity', Comptes Rendus de l'Académie des Sciences-Series IIB, Vol. 329, No. 6, pp.429-434.

Lebon, F. and Rizzoni, R. (2008) 'Asymptotic study of a soft thin layer: the non convex case', Mechanics of Advanced Materials and Structures, Vol. 15, No. 1, pp.12-20.

Lebon, F. and Rizzoni, R. (2010) 'Asymptotic analysis of a thin interface: the case involving similar rigidity', International Journal of Engineering Science, Vol. 48, No. 5, pp.473-486.

Lebon, F. and Rizzoni, R. (2011) 'Asymptotic behavior of a hard thin linear interphase: an energy approach', International Journal of Solids and Structures, Vol. 48, pp.441-449.

Mauge, C. and Kachanov, M. (1994) 'Effective elastic properties of an anisotropic material with arbitrarily oriented interacting cracks', Journal of the Mechanics and Physics of Solids, Vol. 42, pp.561-584.

Milani, G., Lourenço, P.B. and Tralli, A. (2006) 'Homogenised limit analysis of masonry walls, Part I: failure surfaces', Computers \& Structures, Vol. 84, Nos. 3-4, pp.166-180.

Pelissou, C. and Lebon, F. (2009) 'Asymptotic modeling of quasi-brittle interfaces', Computers \& Structures, Vol. 87, Nos. 19-20, pp.1216-1223. 
Raffa, M.L. (2015) Micromechanical Modeling of Imperfect Interfaces and Applications. PhD Thesis, University of Rome "Tor Vergata" and Aix-Marseille University.

Raffa, M. L., Lebon, F. and Rizzoni, R. (submitted). 'Derivation of a model of imperfect interface with finite strains and damage by asymptotic techniques. Application to masonry structures', Meccanica.

Raffa, M.L., Lebon, F. and Rizzoni, R. (2016a) 'On modeling brick/mortar interface via a St. VenantKirchhoff orthotropic soft-interface. Part I: theory', International Journal of Masonry Research and Innovation, Vol. 1, No. 2, pp.142-164.

Raffa, M.L., Lebon, F. and Vairo, G. (2016b) 'Normal and tangential stiffnesses of rough surfaces in contact via an imperfect interface model', International Journal of Solids and Structures, Vol. 87, pp.245-253.

Rekik, A. and Lebon, F. (2010) 'Identification of the representative crack length evolution in a multilevel interface model for quasi-brittle masonry', International Journal of Solids and Structures, Vol. 47, Nos. 22-23, pp.3011-3021.

Rekik, A. and Lebon F. (2012) 'Homogenization methods for interface modeling in damaged masonry', Advances in Engineering Software, Vol. 46, No. 1, pp.35-42.

Rizzoni, R. and Lebon, F. (2013) 'Imperfect interfaces as asymptotic models of thin curved elastic adhesive interphases', Mechanics Research Communications, Vol. 51, pp.39-50.

Rizzoni, R., Dumont, S., Lebon, F. and Sacco, E. (2014) 'Higher order model for soft and hard elastic interfaces', International Journal of Solids and Structures, Vol. 51, Nos. 23-24, pp.4137-4148.

Rizzoni, R., Dumont, S. and Lebon, F. (2017) 'On Saint Venant - Kirchhoff imperfect interfaces', International Journal of Non-Linear Mechanics, Vol. 89, pp.101-115.

Sanchez-Palencia, E. (1980) Non Homogeneous Materials and Vibration Theory, Lecture Notes in Physics, Springer, Berlin, 127.

Scimemi, G., Giambanco, G. and Spada, A. (2014) 'The interphase model applied to the analysis of masonry structures', Computer Methods in Applied Mechanics and Engineering, Vol. 279, pp.66-85

Sevostianov, I. and Kachanov, M. (2013) 'Non-interaction approximation in the problem of effective properties', in Kachanov, M. and Sevostianov, I. (Eds.): Effective Properties of Heterogeneous Materials. Vol. 193 of Solid Mechanics and Its Applications. Springer, Netherlands, pp.1-95.

Tsukrov, I. and Kachanov, M. (2000) 'Effective moduli of an anisotropic material with elliptical holes of arbitrary orientational distribution', International Journal of Solids and Structures, Vol. 37 , No. 41, pp.5919-5941.

Vermeltfoort, A.T. and van Schijndel, A.W.M. (2008) 'Modelling of Lintel-Masonry interaction using COMSOL', Proceedings of the COMSOL Conference, Hannover, Germany, 04-06.11.2008.

Vermeltfoort, A.T. and van Schijndel, A.W.M. (2010) 'Stress distribution in masonry walls, loaded in plane, simulated with COMSOL', Proceedings of the COMSOL Conference, Paris, France, 17-19.11.2010.

Vermeltfoort, A.T. and van Schijndel, A.W.M. (2013) 'COMSOL simulations of cracking in point loaded masonry with randomly distributed material properties', Proceedings of the COMSOL Conference, Rotterdam, Netherlands, 23-25.10.2013. 